\title{
ADDENDUM TO THE PAPER ON PARTIALLY STABLE ALGEBRAS
}

BY

\section{A. A. ALBERT}

I regret to announce that there is a serious error in my paper in these Transactions, volume 84 , pp. $430-443$. The error was discovered by Louis Kokoris who found that on line 8 of page 434 the expression given as $4[g(b z)](a z)$ should have been $4[g(a z)](b z)$. As a consequence the computation of $P(z, g, a z, b)$ yields nothing, the proof of formula (30) is not valid, and the important Lemma 9 is not proved. Thus the paper does not give a proof of its major result stated as Theorem 1.

Nevertheless, the theorems of the paper are all correct and we shall provide a revision of the proof here. This revised proof has been checked by Louis Kokoris to whom the author wishes to express his great thanks.

We observe first that the equation

$$
g S_{a b}=g S_{a} S_{b}-(w a)\left[z \phi_{g}(b)\right]
$$

occurs before the error and is correct. The results of $\$ 4$ on pages 434 and 435 depend on Lemma 9, and their use must be postponed until we apply them in a new Lemma 25. Equation (41) is correct as proved and will become a part of a corrected version of Lemma 17. The following result will be used here and is proved correctly in the paper.

Lemma 14. Let $a$ and $b$ be in $\mathfrak{B}$ and $g$ be in (5). Then

$$
g[(w a)(b z)]=b \phi_{g}(a)-\phi_{g S b}(a) .
$$

Lemma 17 uses Theorem 1 and so needs revision. The computation of $P(w a, b z, c, w)$ of its proof actually yields $4 a(b c)-3 b(c a)-c(a b)=\phi_{h}(a)$ $-4 \phi_{o}(c)$. The interchange of $a$ and $c$ implies the interchange of $g$ and $h$ and results in the formula $4 c(b a)-3 b(c a)-a(c b)=\phi_{g}(c)-4 \phi_{h}(a)$. The quantity $\phi_{h}(a)$ can now be eliminated and we obtain the second equation in a relation which we shall number (41R). The proof of the first relation is exactly as in the paper.

LEMma 17R. Let $g=(w a)(b z)$, where $a$ and $b$ are in $\mathfrak{B}$. Then

$$
f_{g}(c)=0, \quad \phi_{g}(c)=(a c) b-a(c b)
$$

for every $c$ of $\mathfrak{B}$.

Since Theorem 1 is still not valid at this point we shall require the following result.

Received by the editors June 26, 1957. 
Lemma A. The algebra $\mathfrak{B}$ is a special Jordan algebra such that $\mathfrak{B}=e \mathfrak{F}+\mathfrak{R}$, where $e$ is the unity element of the algebra $\mathfrak{A}$, and $\mathfrak{R}$ is the radical of $\mathfrak{B}$.

For Lemma 2 states that the algebra $\mathfrak{B}$ is isomorphic to the algebra $\mathfrak{A}_{u}(1)=\mathfrak{B} u$. It is also known ${ }^{(1)}$ that the mapping $a_{1} \rightarrow S_{1 / 2}\left(a_{1}\right)$, defined by the multiplication $x_{1 / 2} a_{1}=x_{1 / 2} S_{1 / 2}\left(a_{1}\right)$ in our $u$-stable case, is a homomorphic mapping of $\mathfrak{B} u$ onto the special Jordan algebra of all of the linear transformations $S_{1 / 2}\left(a_{1}\right)$ where $a_{1}$ ranges over all elements of $\mathfrak{B} u$. These elements all have the form $a_{1}=a u$ for $a$ in $\mathfrak{B}$, and if $S_{1 / 2}\left(a_{1}\right)=0$ then $w S_{1 / 2}\left(a_{1}\right)=w(a u)=0$. However, Lemma 3 states that $w(a u)=2^{-1}(w a)$. Hence the kernel of our homomorphism consists of all elements $a u$ such that $w a=0, w(w a)=a=0$. It follows immediately that $\mathfrak{B}$ is a special Jordan algebra. The basic assumption that $\mathfrak{A}$ has degree two implies that the only idempotent of $\mathfrak{B} u$ is $u$, the only idempotent of $\mathfrak{B}$ is its unity element $e$. It is then known $\left({ }^{2}\right)$ that $\mathfrak{B}=e \mathfrak{F}+\mathfrak{N}$ as desired.

We are now able to use Lemma $17 \mathrm{R}$, Lemma A, and Lemma 4 to derive the following result.

Lemma B. The inclusion relation $[(w \mathfrak{B})(\mathfrak{B} z)](\mathfrak{B} z) \subseteq w \mathfrak{R}$ holds.

For, we can always write every element $a$ of $\mathfrak{B}$ in the form $a=\alpha e+c$ where $\alpha$ is in $\mathfrak{F}$ and $c$ is in $\Re$. Since $w(b z)=(w a) z=0$ for every $a$ and $b$ of $\mathfrak{B}$ we also write $b$ in the form $b=\beta e+d$ with $d$ in $\mathfrak{R}$ and have $(w a)(b z)=(w c)(d z)$, that is,

$$
(w \mathfrak{B})(\mathfrak{B} z)=(w \mathfrak{R})(\mathfrak{\Re} z) .
$$

But if $g=(w a)(b z)$ we use $(41 \mathrm{R})$ to see that $[(w a)(b z)](t z)=-w \phi_{g}(t)$ where $\phi_{g}(t)=(a t) b-a(t b)$. By $(1 \mathrm{~A})$ we can take both $a$ and $b$ to be in $\mathfrak{R}, \phi_{g}(t)$ is in $\mathfrak{A}$ and our proof is complete.

Lemmas 18 and 19 of our original version state that $[w(b c)](a z)$ $=b[(w c)(a z)]+c[(w b)(a z)]$ and that consequently

$$
f_{\theta}(a)[(w a)(b z)]=0
$$

for every $a$ and $b$ of $\mathfrak{B}$. However, we cannot use Lemma 16 to conclude that $(w \mathfrak{B})(\mathfrak{B} z)=0$ and we shall, in fact, be forced to delete Lemma 16. The proof of the relation

$$
\left[w f_{\theta}(a)\right](z b)+\left[w f_{o}(b)\right](z a)=0
$$

is correct and this relation is used later. We now use our results to obtain a corrected proof of the following lemma.

LEMMA 20. Let there exist a nonsingular element $c=f_{g}(a)$ for some $g$ in $(5)$ and $a$ in $\mathfrak{B}$. Then $(w B)(B z)=0$.

(1) See Lemma 1 of $A$ theory of power-associative commutative algebras, these Transactions, vol. 69 (1950) pp. 503-527.

(2) Ibid., Theorem 2. 
For (45) is equivalent to $(w c)(a z)=0$ if $c=f_{g}(a)$. Lemma 18 then implies that in this case $[w(b c)](a z)=c[(w b)(a z)]$. However, (10) states that $(w b)(a z)$ $=-(w a)(b z)$ and so (47) implies that $[w(b c)](a z)=0$. If $c$ is any nonsingular element of the special Jordan algebra $\mathfrak{B}$ we can write $c=\gamma e+d$ where $d$ is in $\mathfrak{N}$ and $\gamma$ is in $\mathfrak{F}$. It is known $\left(^{3}\right)$ that the corresponding right multiplication $R_{d}$ of the algebra $\mathfrak{B}$ is nilpotent and it follows that the right multiplication $R_{c}=\gamma I+R_{d}$ is nonsingular. Hence the equation $b c=t$ has a solution $b$ in $\mathfrak{B}$ for every $t$ of $\mathfrak{B}$. It follows that our assumption that $c=f_{g}(a)$ is nonsingular implies that $(w t)(a z)=0$ for every $t$ of $\mathfrak{B}$ and every $a$ of $\mathfrak{B}$ such that $f_{g}(a)$ is nonsingular. It remains to consider the singular elements $f_{g}(m)$. The sum $f_{g}(m)+f_{g}(a)=f_{g}(a+m)$ is then nonsingular, that is, is not in the radical $\mathfrak{R}$. Then our proof implies that $(w t)[(a+m) z]=0=(w t)(a z)+(w t)(m z)=(w t)(m z)$ as desired, and we have completed a proof of the relation $(w \mathfrak{B})(\mathfrak{B z})=0$ in the case of a nonsingular value of the function $f_{g}(a)$.

We are now able to combine our results so as to restore the validity of the first of our basic theorems.

Lemma C. Let a simple commutative power-associative algebra $\mathfrak{A}$ of degree two contain an element $g$ of $B$ and an element $a$ of $\mathfrak{B}$ such that $f_{g}(a)$ is nonsingular. Then Lemmas 9, 10,11, 12, 13, and 15 are all correct and Theorem 1 is correct.

For Lemma 20 implies that the relation (29) reduces to $S_{a b}=S_{a} S_{b}=S_{b} S_{a}$ and this and Lemma 20 give Lemma 9 completely. Thus all of the results of $\S 4$ are now correct.

Lemma $C$ reduces our study to what we have called the "singular case," and to the possible case where every $f_{g}(a)$ is singular but some $\phi_{g}(a)$ is nonsingular. We shall actually be able to show that the latter case cannot occur and indeed that every $\phi_{g}(a)$ is in $\mathfrak{\Re}$. Our first new result in this direction follows.

Lemma D. Let $a, b$, and $c$ be in $\mathfrak{B}$ so that $g=(w a)(b z)$ and $h=(w a)(c z)$ are in G. Then gh is in $\mathfrak{R}$.

By (1A) we can assume that $a, b$, and $c$ are all in $\mathfrak{R}$. Compute $P(w a, w a$, $b z, c z)$ to see that $8 g h+4 a^{2}(b c)=2(w a)[g(c z)+h(b z)+(w a)(b c)]+(b z)\left[a^{2}(c z)\right.$ $+2 h(w a)]+(c z)\left[a^{2}(b z)+2 g(w a)\right]$. Then

$$
\begin{aligned}
8 g h= & -4 a^{2}(b c)+2(w a)\left[-w \phi_{g}(c)-w \phi_{h}(b)+w \cdot a(b c)\right]+b\left(a^{2} c\right) \\
& +2 b\left[\phi_{h}(a)+z f_{h}(a)\right]+c\left(a^{2} b\right)+2 c\left[\phi_{g}(a)+z f_{g}(a)\right] .
\end{aligned}
$$

Since $a, b$, and $c$ are all in the radical $\mathfrak{R}$ of $\mathfrak{B}$ all terms on the right side of this equation are in $\mathfrak{l}+\mathfrak{N} z$. But $g h$ is in $\mathfrak{B}$ and hence is in $\mathfrak{R}$.

(3) See Theorem 1 of the author's $A$ structure theory for Jordan algebras, Ann. of Math. vol. 48 (19+7) pp. 546-567. 
We now use the relation (42) in the form

$$
h[(w a)(t z)]=t \phi_{h}(a)-\phi_{h S_{t}}(a),
$$

where $h$ is any element of $\mathcal{G}$, and $a$ and $t$ are arbitrary elements of $\mathfrak{B}$. Assume that

$$
h=(w a)(d z)
$$

for the same $a$ and some $d$ in $\mathfrak{B}$ and use Lemma $\mathrm{B}$ to see that $\phi_{h}(a)$ is in $\mathfrak{N}$ and so $t \phi_{h}(a)$ is in $\Re$. By Lemma D we know that the left member of $(2 \mathrm{~A})$ is in $\mathfrak{N}$. Thus (2A) implies the following result.

Lemma E. Let $a, d$, and $t$ be any elements of $\mathfrak{N}$ and $h=(w a)(d z)$. Then $\phi_{h S_{t}}(a)$ is in $\mathfrak{R}$.

There is a minor error in the statement of Lemma 21 which actually does not affect the argument but which should be corrected as follows. We compute $P(w a, z, g, g)$ and see that

$$
\begin{aligned}
0 & =2 g[(w a) g \cdot z]+(w a)\left(g^{2} z\right)=2 g\left[f_{\theta}(a) \cdot z+\phi_{g}(a)\right]+(w a)\left(g^{2} z\right) \\
& =-2 w\left\{f_{g}\left[\phi_{g}(a)\right]+\phi_{g}\left[f_{g}(a)\right]\right\}+2 g S_{c}+(w a)\left(g^{2} z\right)
\end{aligned}
$$

where $c=\phi_{0}(a)$. This yields the corrected version of Lemma 21 which we now state.

Lemma 21R. Let a be in $\mathfrak{B}, g$ be in $\mathfrak{B}$, and $c=\phi_{g}(a)$. Then

$$
2 g S_{c}=-(w a)\left(g^{2} z\right), \quad f_{o}\left[\phi_{g}(a)\right]+\phi_{g}\left[f_{o}(a)\right]=0 .
$$

We have now obtained the key elements in a proof of the singularity of the function $\phi_{g}(a)$.

LEMMA F. The elements $\phi_{0}(a)$ are in $\mathfrak{N}$ for every $g$ of $(\$)$ and a of $\mathfrak{B}$.

For assume that there does exist an element $g$ of $(s)$ and an element $a$ of $\mathfrak{B}$ such that $c=\phi_{\theta}(a)$ is nonsingular. By $(49 \mathrm{R})$ we have

$$
h=g S_{c}=(w a)(d z)
$$

where $d=-1 / 2 g^{2}$ is in $\mathfrak{B}$. We now use (29) to see that if $t=c^{-1}$ then $g S_{c t}$ $=g S_{e}=g$, and so

$$
g S_{c} S_{t}=h S_{t}=g-(w c)\left[z \phi_{\theta}(t)\right]
$$

Hence

$$
g=h S_{t}+k
$$

where $k$ is in $(w \mathfrak{B})(\mathfrak{B z})$. By $(4 \mathrm{~A})$ and Lemma $\mathrm{E}$ we know that $\phi_{h S_{t}}(a)$ is in $\mathfrak{N}$. By Lemma $\mathrm{B}$ we know that $\phi_{k}(a)$ is in $\Re$. But then (6A) implies that $\phi_{v}(a)$ $=\phi_{h S_{t}}(a)+\phi_{k}(a)=c$ is in $\mathfrak{N}$, contrary to hypothesis. 
We are now ready to turn to the discussion of the singular case of Section 6. The proof there makes some slight and unnecessary use of Lemma 9 and we correct the argument as follows. Note that Lemma 9 cannot be used since we have only proved its validity in the nonsingular case. We observe that

$$
\mathfrak{A}=\mathfrak{B}+\mathfrak{B} z+w \mathfrak{B}+\mathfrak{B}
$$

and we now write

$$
\mathfrak{M}=\mathfrak{R}+\mathfrak{R z},
$$

so that $\mathfrak{M}$ is the radical of the Jordan algebra $\mathfrak{E}=\mathfrak{A}_{u}(1)+\mathfrak{A}_{u}(0)=\mathfrak{B}+\mathfrak{B} z$. Then the combination of the hypothesis that every $f_{g}(a)$ is in $\mathfrak{R}$ and Lemma $\mathrm{F}$ is equivalent to the inclusion relations

$$
(w \mathfrak{B}) \mathfrak{B} \subseteq \mathfrak{M}, \quad \mathfrak{B}(\mathfrak{B z}) \subseteq w \mathfrak{N} .
$$

Define (5* to be the vector spanned by all elements of the form $g S_{a}$ for $g$ in (B) and $a$ in $\mathfrak{N}$. Then Lemma $\mathrm{F}$ implies that

$$
\mathfrak{S M} \subseteq \mathfrak{B}^{*}+w \mathfrak{N}, \quad \mathfrak{B}^{*} \mathfrak{B}=\mathfrak{B}^{*}+\mathfrak{B}^{*} \mathfrak{R} \subseteq \mathfrak{B}^{*} .
$$

Also Lemma $F$ and (42) imply that

$$
\mathfrak{B S}[(w \mathfrak{B})(\mathfrak{B} z)] \subseteq \mathfrak{N} .
$$

Then (29) implies that, if we write $x \equiv y$ for $x$ and $y$ in $\mathfrak{B}$ whenever $x-y$ is in $\mathfrak{N}$, we have the property

$$
\left(g S_{a b}\right) h \equiv\left(g S_{a} S_{b}\right) h
$$

for every $g$ and $h$ of $B$ and $a$ and $b$ of $\mathfrak{N}$. We now have all of the results of (55)-(60), the argument which forms the last paragraph of page 440 is valid, and the relation

$$
\text { (3)(S) } \subseteq \mathfrak{N}
$$

is correct.

We are now able to prove that the space

$$
\mathfrak{S}=w \mathfrak{N}+\left(\mathfrak{B}^{*}+(w \mathfrak{B})(\mathfrak{B} z)+\mathfrak{M}\right.
$$

is an ideal of $\mathfrak{R}$. Indeed $\mathfrak{A M}=\mathfrak{M M}+(w \mathfrak{B}) \mathfrak{M}+\mathfrak{H M} \subseteq \mathfrak{M}+w \mathfrak{R}+(w \mathfrak{B})(\mathfrak{B} z)$ $+\mathfrak{B}^{*} \subseteq \mathfrak{S}$. Also $\mathfrak{\Re}(w \mathfrak{N})=\mathfrak{B}(w \mathfrak{N})+(\mathfrak{B} z)(w \mathfrak{N})+\mathfrak{B}(w \mathfrak{N})+(w \mathfrak{B})(w \mathfrak{R}) \subseteq w \mathfrak{N}$

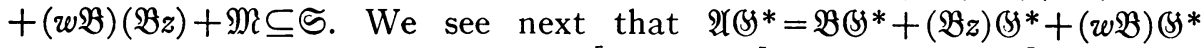
$+\mathfrak{S B S}^{*} \subseteq \mathfrak{S}^{*}+w \mathfrak{N}+\mathfrak{M} \subseteq \subseteq$, and that $\mathfrak{A}[(w \mathfrak{B})(\mathfrak{B z})]=(w \mathfrak{B})(\mathfrak{B z})+\mathfrak{N}[(w \mathfrak{B})(\mathfrak{B} z)]$ $+(\mathfrak{B z})[(w \mathfrak{B})(\mathfrak{B} z)]+(w \mathfrak{B})[(w \mathfrak{B})(\mathfrak{B z})]+(w \mathfrak{B}) \mathfrak{S} \subseteq(w \mathfrak{B})(\mathfrak{B} z)+(\mathfrak{B} *+w \mathfrak{N}+w \mathfrak{N}$ $+\mathfrak{M} \subseteq \subseteq$ and our proof that $\subseteq$ is an ideal of $\mathfrak{A}$ is complete. Since $\subseteq$ does not contain the idempotent $e$ the hypothesis that $\mathfrak{R}$ is simple implies that $\mathfrak{M}=0$, $\mathfrak{B}=e \mathfrak{F}$, and so $\mathfrak{A}$ is actually a Jordan algebra in this case. 
This completes our revision but we take this opportunity to correct the following misprints:

p. 431 , line -4 . Should read $w\left[(a b) g-w \phi_{g}(a) \cdot(b z)-w \phi_{g}(b) \cdot(a z)\right]$.

p. 432 , line 3 . Delete $-4 a f_{\theta}(b)$ at end of sentence.

p. 432 , formula (13). Replace $\phi_{g}(a)$ by $\phi_{g}(a b)$.

p. 432 , line 3 below (13). Replace first - by + .

p. 432 , line 4 below (13). Replace first - by + .

p. 433 , line 11 . Replace last $g S_{a} S_{b}$ by $g S_{b} S_{a}$.

p. 435 , Lemma 13 . Replace $M$ by $\mathfrak{M}$.

p. 436 , line below (44). Replace $\mathfrak{A}$ by $\mathfrak{S}$.

p. 436 , line 7 below (44). Add $+(a b)(z c)$ at end of line.

p. 436, line 8 below (44). Add $+(a b)(z c)$ before "Since."

p. 436 , line 10 below (44). Add $+(a b)(z c)$ before "is in."

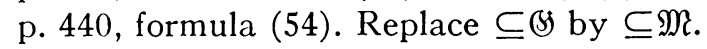

YaLe University $\left({ }^{4}\right)$

New Haven, Conn.

(4) The work on this paper was sponsored by the Office of Ordnance Research, United States Army, under Contract DA-19-059-ORD-2329. 